\title{
Chronic sympathetic driven hypertension promotes atherosclerosis by enhancing hematopoiesis
}

Haematologica 2018

Volume 104(3):456-467

\section{Correspondence:}

ANDREW J. MURPHY

andrew.murphy@baker.edu.au

ANNAS AL-SHAREA

annas.al-sharea@baker.edu.au

Received: March 7, 2018.

Accepted: October 22, 2018.

Pre-published: October 25, 2018.

doi:10.3324/haematol.2018.192898

Check the online version for the most updated information on this article, online supplements, and information on authorship \& disclosures: www.haematologica.org/content/104/3/456

\section{(C2019 Ferrata Storti Foundation}

Material published in Haematologica is covered by copyright. All rights are reserved to the Ferrata Storti Foundation. Use of published material is allowed under the following terms and conditions:

https://creativecommons.org/licenses/by-nc/4.0/legalcode. Copies of published material are allowed for personal or internal use. Sharing published material for non-commercial purposes is subject to the following conditions:

https://creativecommons.org/licenses/by-nc/4.0/leǵalcode, sect. 3. Reproducing and sharing published material for commercial purposes is not allowed without permission in writing from the publisher.

\author{
Annas Al-Sharea,${ }^{1 *}$ Man K. S. Lee, ${ }^{1}$ Alexandra Whillas,${ }^{1}$ Danielle L. Michell,${ }^{1,2}$ \\ Waled A. Shihata, ${ }^{1,3}$ Alyce J. Nicholls, ${ }^{4}$ Olivia D. Cooney, ${ }^{1}$ Michael J. \\ Kraakman, ${ }^{1,5}$ Camilla Bertuzzo Veiga, ${ }^{1}$ Ann-Maree Jefferis, ${ }^{3}$ Kristy Jackson, ${ }^{6}$ \\ Prabhakara R. Nagareddy, ${ }^{7}$ Gavin Lambert, ${ }^{8,9}$ Connie H. Y. Wong, ${ }^{4}$ \\ Karen L. Andrews, ${ }^{3}$ Geoff A. Head, ${ }^{6}$ Jaye Chin-Dusting ${ }^{3}$ and \\ Andrew J. Murphy ${ }^{1,10^{*}}$
}

${ }^{1}$ Haematopoiesis and Leukocyte Biology Laboratory, Division of Immunometabolism, Baker Heart and Diabetes Institute, Melbourne, VIC, Australia; ${ }^{2}$ Department of Medicine, Vanderbilt University School of Medicine, Nashville, TN, USA; ${ }^{3}$ Department of Pharmacology, Monash University, Clayton, VIC, Australia; ${ }^{4}$ Monash University, Melbourne, VIC, Australia; ${ }^{5}$ Naomi Berrie Diabetes Center and Department of Medicine, Columbia University, New York, NY, USA; ${ }^{6}$ Neuropharmacology Laboratory, Division of Hypertension and Cardiac Disease, Baker Heart and Diabetes Institute, Melbourne, VIC, Australia; 'Department of Nutrition Sciences, University of Alabama at Birmingham, AL, USA; ${ }^{~}$ Human Neurotransmitters Laboratory, Division of Hypertension and Cardiac Disease, Baker Heart and Diabetes Institute, Melbourne, VIC, Australia; ${ }^{9}$ Iverson Health Innovation Research Institute, Swinburne University of Technology, Hawthorn, VIC, Australia; ${ }^{10}$ Department of Immunology, Monash University, Melbourne, VIC, Australia

*Corresponding Authors

\section{ABSTRACT}

$\tau$ T ypertension is a major, independent risk factor for atherosclerotic cardiovascular disease. However, this pathology can arise through multiple pathways, which could influence vascular disease through distinct mechanisms. An overactive sympathetic nervous system is a dominant pathway that can precipitate in elevated blood pressure. We aimed to determine how the sympathetic nervous system directly promotes atherosclerosis in the setting of hypertension. We used a mouse model of sympathetic nervous system-driven hypertension on the atherosclerotic-prone apolipoprotein E-deficient background. When mice were placed on a western type diet for 16 weeks, we showed the evolution of unstable atherosclerotic lesions. Fortuitously, the changes in lesion composition were independent of endothelial dysfunction, allowing for the discovery of alternative mechanisms. With the use of flow cytometry and bone marrow imaging, we found that sympathetic activation caused deterioration of the hematopoietic stem and progenitor cell niche in the bone marrow, promoting the liberation of these cells into the circulation and extramedullary hematopoiesis in the spleen. Specifically, sympathetic activation reduced the abundance of key hematopoietic stem and progenitor cell niche cells, sinusoidal endothelial cells and osteoblasts. Additionally, sympathetic bone marrow activity prompted neutrophils to secrete proteases to cleave the hematopoietic stem and progenitor cell surface receptor CXCR4. All these effects could be reversed using the $\beta$-blocker propranolol during the feeding period. These findings suggest that elevated blood pressure driven by the sympathetic nervous system can influence mechanisms that modulate the hematopoietic system to promote atherosclerosis and contribute to cardiovascular events.

\section{Introduction}

Hypertension is a major, independent risk factor for atherosclerotic cardiovascular disease (CVD). ${ }^{1}$ As the pathophysiology of hypertension is both complex and multifactorial, the direct mechanism(s) that ultimately contribute to CVD remain 
unclear. $^{2}$ The most frequently targeted pathway in reducing blood pressure is the renin-angiotensin system (RAS). The contribution of the RAS to hypertension and atherosclerosis is not exclusive, as angiotensin II (AngII) can also accelerate atherogenesis independent of hypertension. ${ }^{3,4}$ Another major determinant of hypertension is an overactive sympathetic nervous system (SNS)., ${ }^{5,6}$ There are indications that autonomic input into the bone marrow (BM) may be altered in the setting of hypertension. ${ }^{7-10}$ However, the mechanisms promoting atherogenesis with SNS activation associated hypertension are not completely elucidated. While there is an overlap in some atherosclerosis promoting mechanisms between the RAS and SNS, a distinct subset of events is also likely to be evoked by the $\mathrm{SNS}$, which requires further investigation.

Atherosclerosis is a disease driven by the infiltration of immune cells, in particular monocytes, into the plaque. ${ }^{11-13}$ It is also well established that the abundance of circulating monocytes predicts cardiovascular (CV) events and is directly linked to atherogenesis. ${ }^{14,15}$ Interestingly, the SNS plays a direct role in regulating the hematopoietic system from which immune cells, including monocytes, arise. ${ }^{16-19}$ In the context of CVD, the mobilization of hematopoietic stem and progenitor cells (HSPCs) from the BM to extramedullary tissues such as the spleen results in the generation of atherogenic monocytes that abundantly enter into the atherosclerotic plaque. ${ }^{20}$ Mobilization of HSPCs can be mediated by sympathetic signaling within the BM, particularly in response to granulocyte-colony stimulating factor (G-CSF). The SNS synergizes with GCSF to promote the breakdown of the HSPC BM microenvironment, which decreases the abundance of key HSPC retention factors and results in the liberation of HSPCs into the circulation. ${ }^{16}$ This pathway has also been shown to be activated following a myocardial infarction (MI). ${ }^{21}$ Sympathetic activation, along with raised G-CSF levels that are observed in apolipoprotein E knockout (Apoer-) mice, caused HSPC mobilization from the BM and homing to the spleen where monocytes were subsequently produced that infiltrated atherosclerotic lesions. Interestingly, this promoted an unstable plaque phenotype, prone to rupturing and thus provides a plausible explanation for primary heart attack survivors being highly prone to a secondary, often fatal, CV event. ${ }^{21,22}$ Importantly, the involvement of the SNS in driving aberrant hematopoiesis is not restricted to complications following a MI, as similarities in other models of stress and ischemic stroke are evident, suggesting this to be a more general mechanism. The augmented hematopoietic response in these pathologies caused by overactivation of the SNS were inhibited by administration of $\beta$-blockers or genetic deletion of $\beta$-adrenergic receptors. ${ }^{21,23-25}$

There appears to be an important role of the SNS in regulating hematopoiesis in acute stressors (i.e., MI, stroke, variable stress). However, it remains unknown if chronic sympathetic activation invokes this same atherogenic process. Thus, it is plausible that chronic sympathetic activation present in some cases of hypertension could play an important role in regulating atherogenesis by altering hematopoiesis. To address this question, we employed the Schlager hypertensive mice which were crossed onto an Apoe- background to produce hypertensive atherosclerosis-prone mice. The Schlager mouse was chosen as it represents a model of hypertension that is almost entirely driven by the SNS, with minimal contribution by the
RAS. ${ }^{26}$ We sought to characterize the contribution of SNS activation associated hypertension to the development of atherosclerosis, with the aim of understanding whether this form of hypertension was also associated with alterations to the hematopoietic system. Moreover, we aimed to investigate whether targeting the SNS could inhibit atherogenesis and, in turn, reveal an additional mechanism of hypertension associated atherosclerosis.

\section{Methods}

Detailed methods are available in Online supplementary Methods.

\section{Animal Models}

Apoe- mice were purchased from Jackson Laboratories and bred at the AMREP Animal centre. To generate hypertensive Apoe mice, $\mathrm{BPH} / 2 \mathrm{~J}$ mice were crossed with Apoe-/- mice to produce $\mathrm{BPH} / 2 \mathrm{~J} \times$ Apoe $^{-r}\left(\mathrm{BPH} / \mathrm{Apoe}^{-1}\right)$ mice. At 6 weeks of age, male Apoe and BPH/ Apoe-/- mice were placed on a western type diet (WTD - SF00-219, Specialty Feeds, Australia; $21 \%$ fat, $0.15 \%$ cholesterol) for 16 weeks. In the first cohort of mice, age-matched mice Apoe-r and $\mathrm{BPH} / \mathrm{Apoe}^{-/}$were placed on a WTD for 16 weeks for end-point analysis. In a second cohort of mice, obtained from a new set of breeders, three groups of aged-matched mice were employed: 1) Apoe-/-, 2) BPH/Apoe ${ }^{-/}$and 3) BPH/ Apoe $e^{-1}$ propranolol (0.5g/L; administered via drinking water for the duration of the WTD feeding). For the propranolol group, mice consumed on average $2.5 \mathrm{ml}$ of water amounting to an average daily dose of 35 $40 \mathrm{mg} / \mathrm{kg} /$ daily of propranolol.

To determine the effect of specific 2-adrenoreceptor blockade on HSPC mobilization and blood pressure we used BPH mice on an $A$ poe $^{+/ t}$ background. The mice were injected daily with ICI118551 (5mg/kg; Abcam, AUS) for 2 weeks.

All animal experiments were approved by the AMREP Animal Ethics Committee and conducted in accordance with the Australian code of practice for the care and use of animals for scientific purposes as stipulated by the National Health and Medical Research Council of Australia. All mice were housed in a normal light and dark cycle and had ad libitum access to food and water. Mice were randomly assigned to treatment and end-point analysis was blinded.

\section{Statistics}

Data are presented as mean \pm SEM (unless stated otherwise) and were analysed using the two-tailed Student $t$-test or One-way ANOVA where appropriate. Analysis of baseline and final blood pressure between strains was performed using a two-way ANOVA with the factors strain (Pstrain) and time (Ptime) followed by a Sidak post-hoc test to account for multiple comparisons. A $P<0.05$ was considered significant. All tests were performed using the Prism software (GraphPad Software, Inc., La Jolla, CA, USA).

\section{Results}

\section{Hypertension associated with chronic sympathetic activation promotes an unstable atherosclerotic phenotype}

To determine the contribution of chronic sympathetic activation in hypertension to atherosclerosis we crossed Schlager hypertensive mice with Apoe ${ }^{-/}$mice $\left(\mathrm{BPH} /\right.$ Apoe $^{-/}$) and compared these to normotensive Apoe mice. Mice were fed a high fat, high cholesterol western type diet (WTD) for 16 weeks. We preferenced this model over con- 
tinual infusions of noradrenaline to allow for circadian fluctuations in blood pressure and heart rate, and to prevent the ongoing immune complications of surgery associated with the use of mini-pumps. Firstly, examining traditional cardiovascular risk factors revealed no change in body weight or cholesterol between the two groups and while blood pressure increased over the feeding period in both strains, the BPH/Apoe $e^{-/}$mice maintained significantly higher blood pressure as measured by tail cuff and radio telemetry (Figure 1, A-C and Online Supplementary Figure S1). The mice were also equally active (Online Supplementary Figure S1). To explore the effect of chronic sympathetic activation associated with hypertension on atherosclerosis, we assessed the atherosclerotic burden in
A

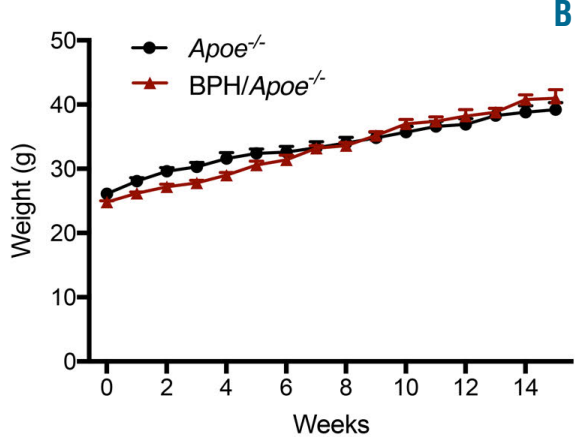

D
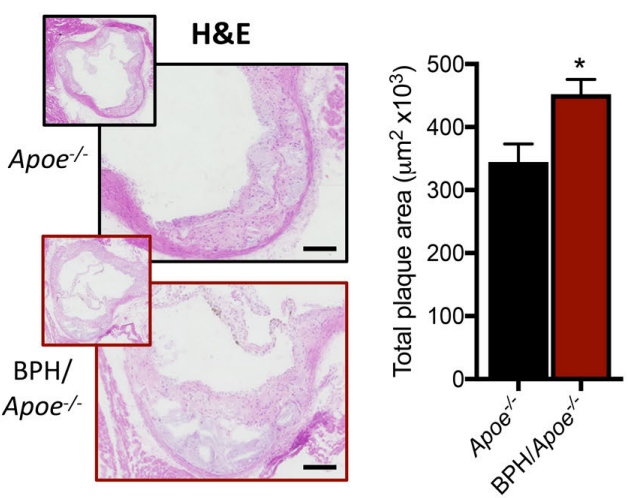

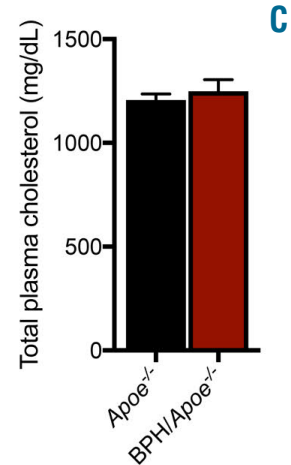

$\mathbf{E}$

C

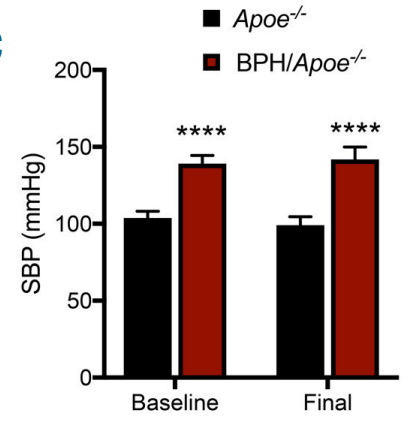

$\mathbf{F}$

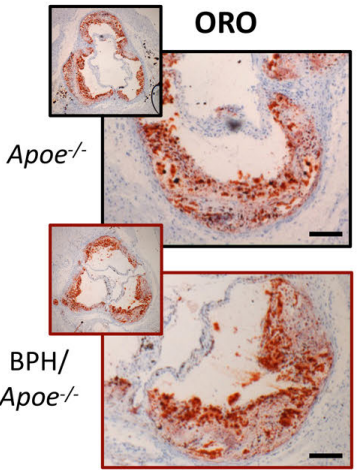

Aortic Arch ORO
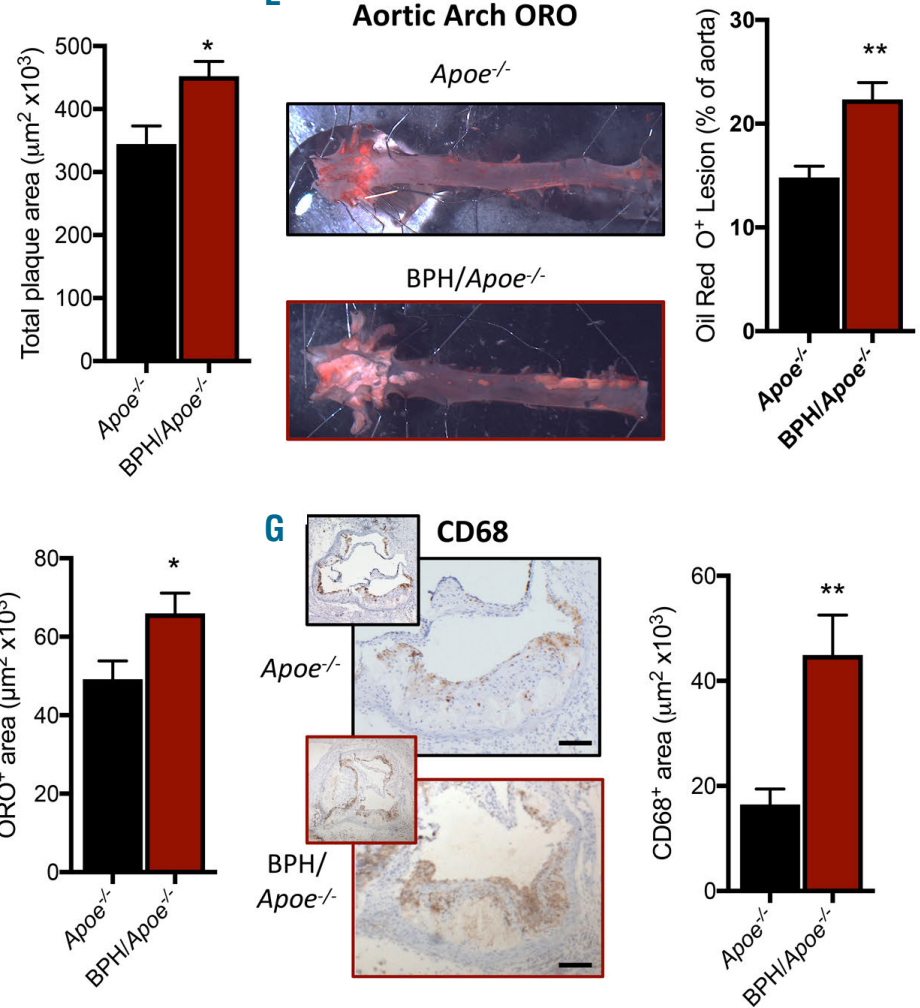

H
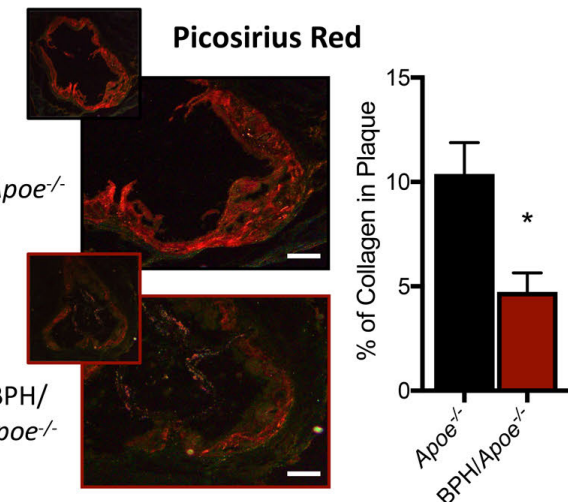

Figure 1. Hypertensive Apoe $\%$ mice have characteristics of unstable atherosclerotic lesions Apoe $\%$ and BPH/Apoe $\%$ mice were fed a WTD for 16 weeks A) Weekly body mass progression. B) Total plasma cholesterol was measured at the end of feeding. C) Systolic blood pressure of mice was measured before and at the end of WTD feeding. D) H\&E staining for plaque size in the proximal aorta and $\mathrm{E}) \mathrm{ORO}^{+}$lesions in the aortic arch were quantified. Proximal aortas were also stained for F) lipid content (ORO), G) macrophages (CD68) and H) collagen (picosirius red). Lesions were imaged at X4 (insets) and zoomed in to view single lesions, scale bar $=100 \mathrm{um}$. Data are presented as mean \pm SEM where $* P<0.05$ and $* * P<0.01$ (Student's $t$-test) $* * * * P<0.0001$ (Two-way ANOVA). A,B,C) $n=5-10, D, E, F) n=8$, G) $n=9, H) n=7-8$. 
the proximal aorta and aortic arch. We observed increases in plaque size between the groups (Figure 1D, E), suggesting that sympathetically driven hypertension may promote accelerated plaque growth. We further explored the lesion characteristics and noted a significant increase in the abundance of lipid within the lesions from the $\mathrm{BPH} /$ Apoe $^{-/}$mice (Figure 1F). A significant increase in plaque macrophages were accompanied by a decrease in plaque collagen in the $\mathrm{BPH} /$ Apoe $^{-/}$mice (Figure $1 \mathrm{G}, \mathrm{H}$ ), suggesting that chronic sympathetic activation was promoting remodeling of lesions in an adverse, unstable man- ner. This plaque phenotype in the $\mathrm{BPH} / \mathrm{Apoe}^{-/}$mice resonates with the findings of Dutta et al. in the context of acute SNS stimulation during a MI. ${ }^{21}$

\section{Hypertensive $\mathrm{Apoe}^{-/-}$mice do not develop endothelial dysfunction}

Endothelial dysfunction is a generally accepted consequence of hypertension. ${ }^{27}$ To determine if the enhanced atherogenesis in $\mathrm{BPH} / A$ poe ${ }^{-/}$mice was the result of endothelial dysfunction we assessed the vascular responses in aortas from the $\mathrm{BPH} / A_{p o e^{-/}}$mice in comparison with

\section{A}

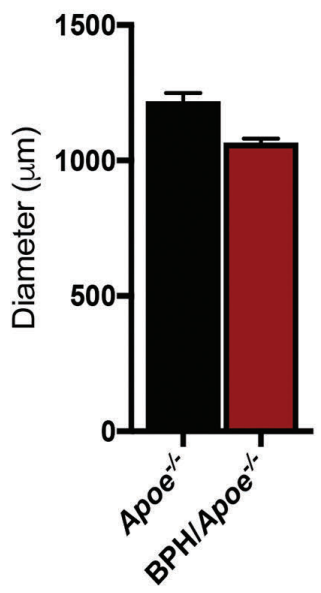

D

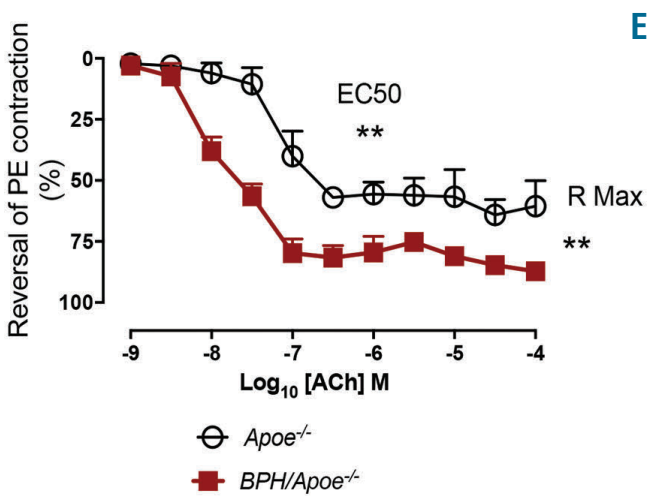

$\mathbf{F}$

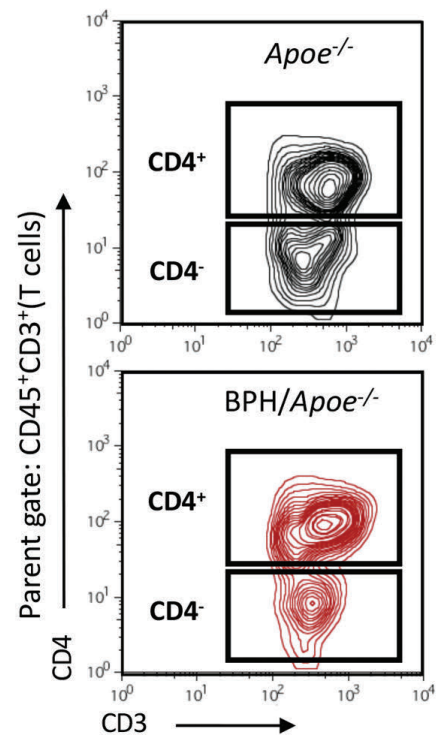

B

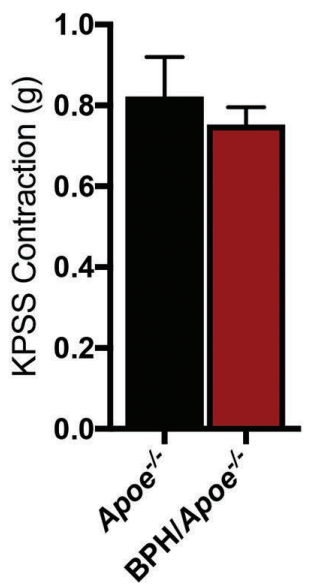

C

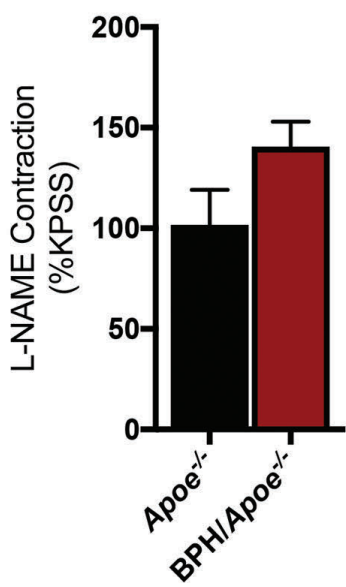

E
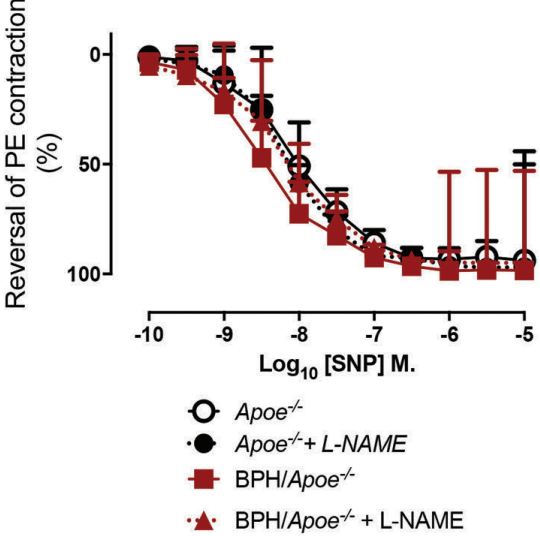

Figure 2. Hypertensive Apoe do not have endothelial dysfunction. Apoe $\%$ and

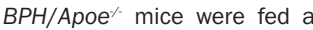
WTD for 16 weeks after which aortas were harvested for ex vivo assessment of endothelial function. Aortas were assessed for A) diameter, B) KPSS and C) L-NAME contraction. Further myograph analyses were preformed to determine aortic relaxation in response to D) ACh and E) SNP with or without L-NAME administration. Aortic T-cell infiltration was assessed by flow cytometry for F) Abundance of CD4 ${ }^{+}$T-helper cells. Data are presented as mean \pm SEM where $* * P<0.01$ (Student's $t$ test). A,B) $n=6-10, C) n=6-8, D$ ) $n=4-6, E, F) n=6$. 
A

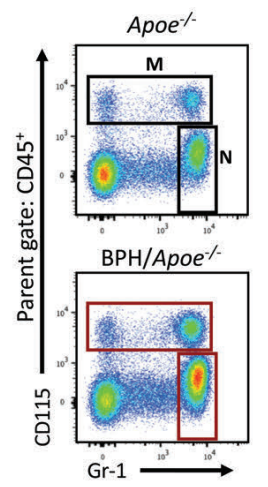

D

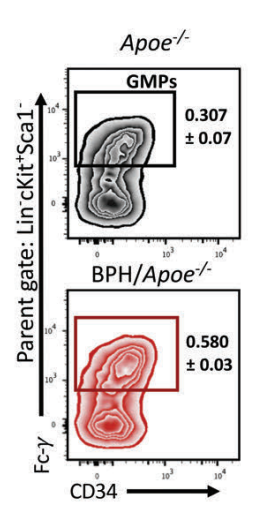

G

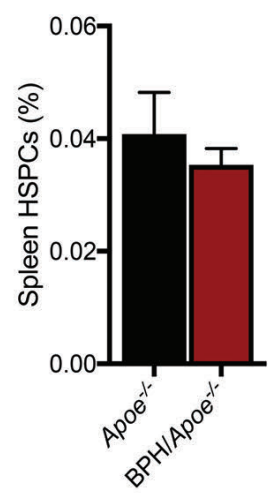

I

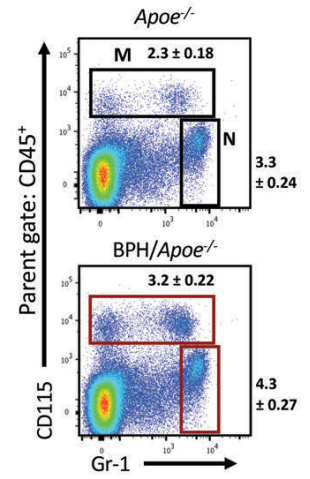

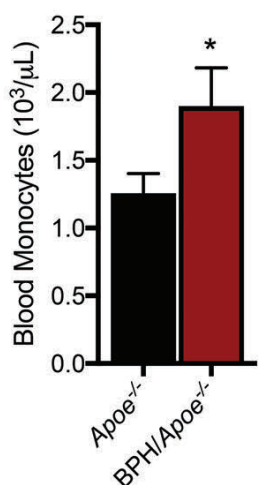
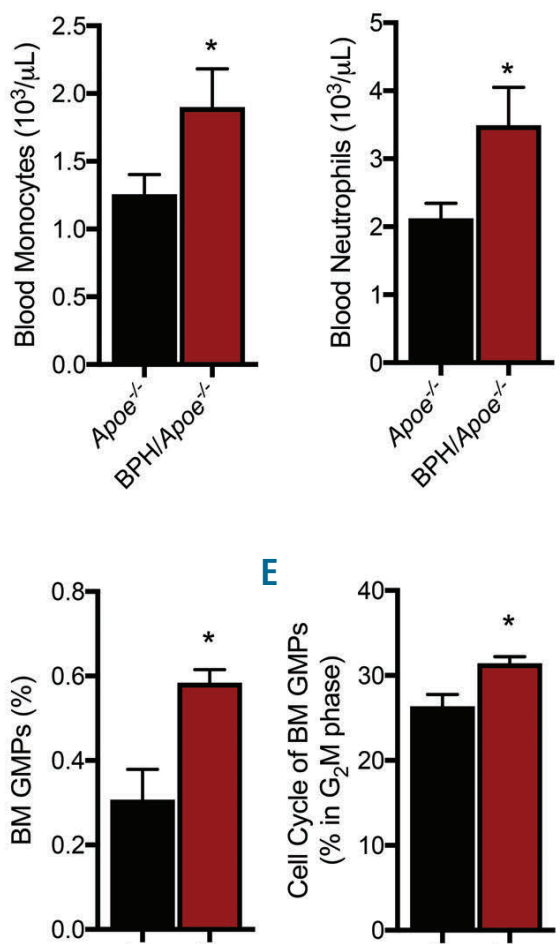

E
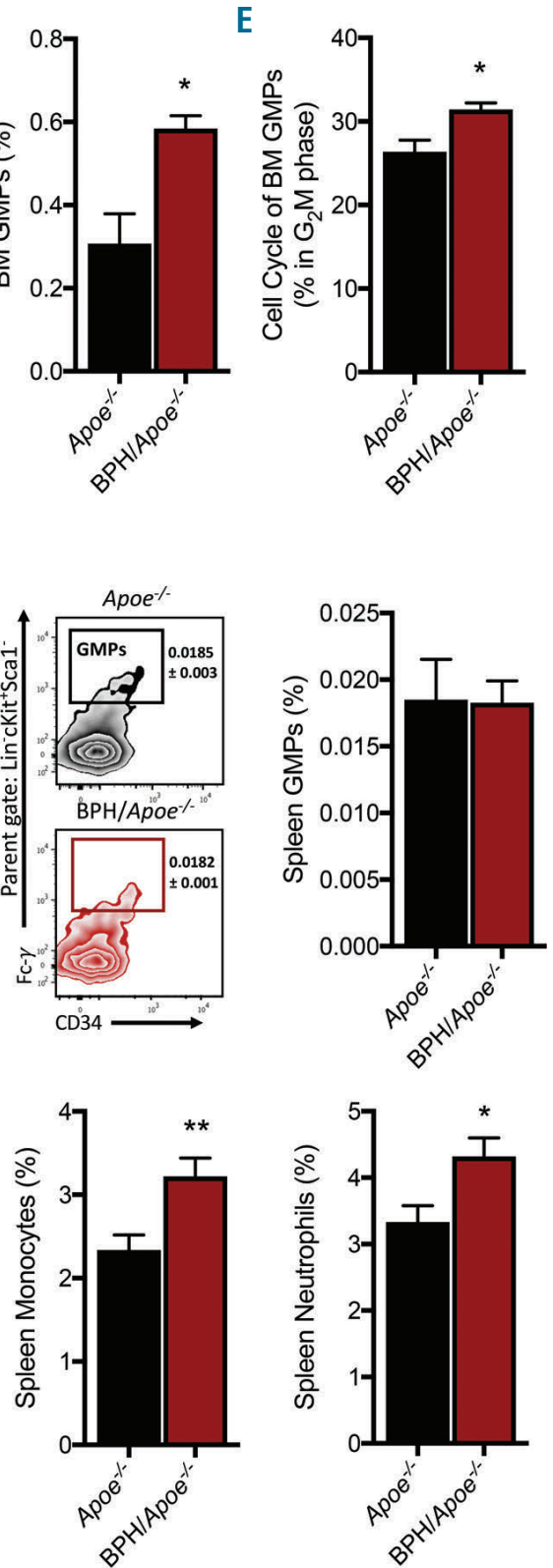

B
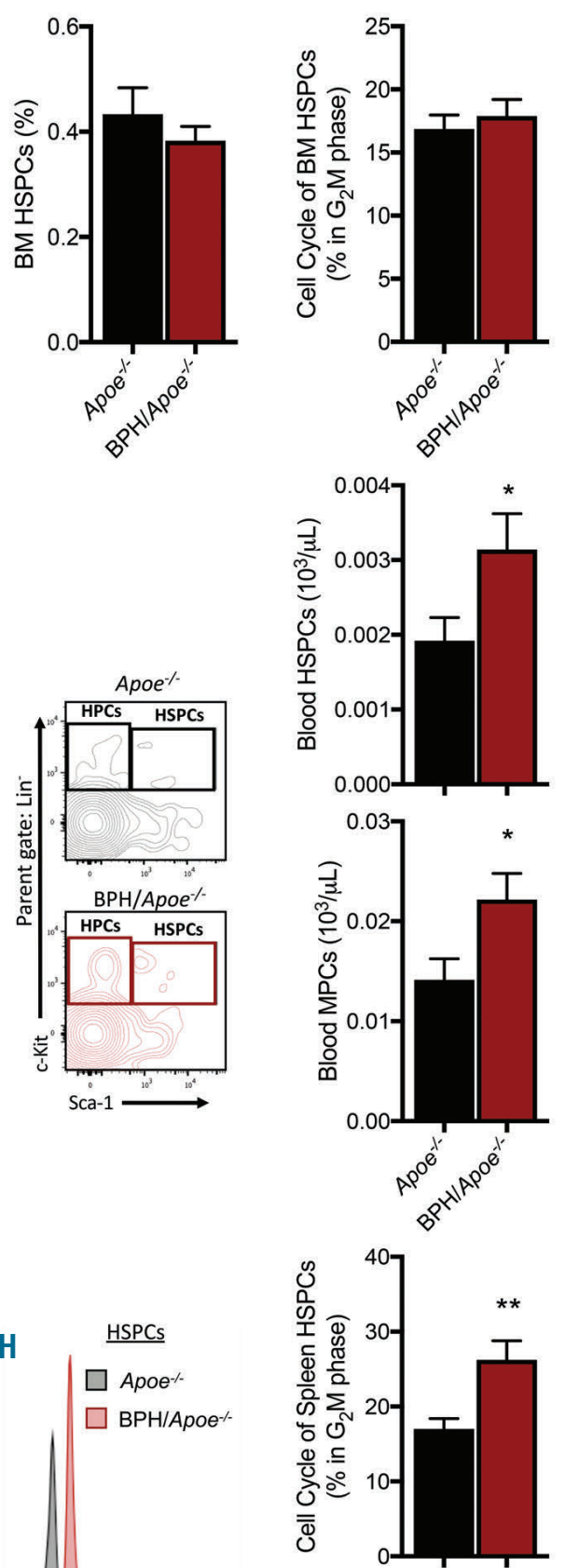

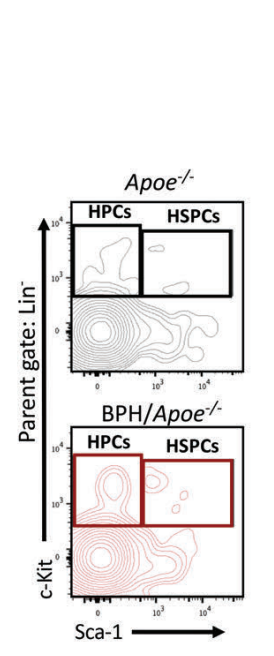

F

H

HSPCS

$\square$ Apoe - -

$\square$ BPH/Apoe
C 
control Apoe $e^{-\mu}$ mice. Firstly, no change in vessel diameter or constrictor responses to a high potassium solution was evident. Nor were there differences in basal nitric oxide (NO) levels when the constriction to L-NAME (L-NGNitroarginine methyl ester) was examined (Figure 2, A-C). These data suggest that alterations in vascular reactivity are not biased by differences in constrictor responses. Surprisingly, endothelium-dependent NO-mediated relaxation in response to acetylcholine (ACh) was worse in the Apoe $e^{-1}$ mice when compared to $\mathrm{BPH} / \mathrm{Apoe}^{-/}$mice (Figure 2D). These differences between the strains were endothelium independent since there were no differences in the constriction and relaxation response to the $\mathrm{NO}$ donor sodium nitroprusside (SNP) in the presence or absence of L-NAME (Figure 2E). To further confirm no decline in vascular function in these mice, we examined the abundance of $\mathrm{T}$ cells, which have been linked to the pathogenesis of hypertension. ${ }^{28}$ We observed no differences in aortic $\mathrm{T}$ cells between the Apoe $e^{-/}$and $\mathrm{BPH} /$ Apoe $^{-/}$mice (Figure $2 \mathrm{~F}$ ). Moreover, there was no difference in the activation state of these $\mathrm{CD}^{+} \mathrm{T}$ cells, as assessed by CD62L expression (MFI; data not shown). These data suggest that the enhanced atherogenesis in the $\mathrm{BPH} / A_{p o e^{-/-}}$mice occurs independently of changes to the endothelium.
A

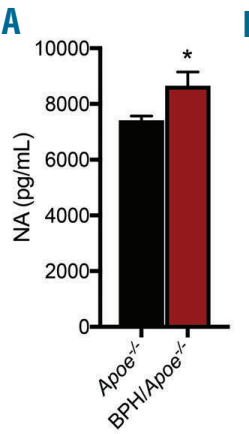

D

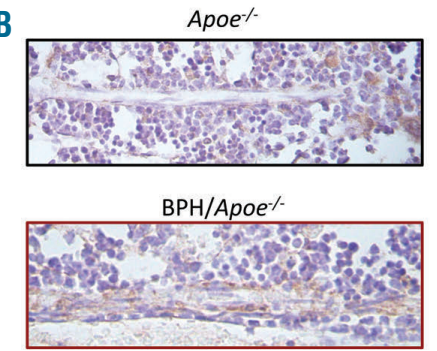

Anti-Tyrosine Hydroxylase
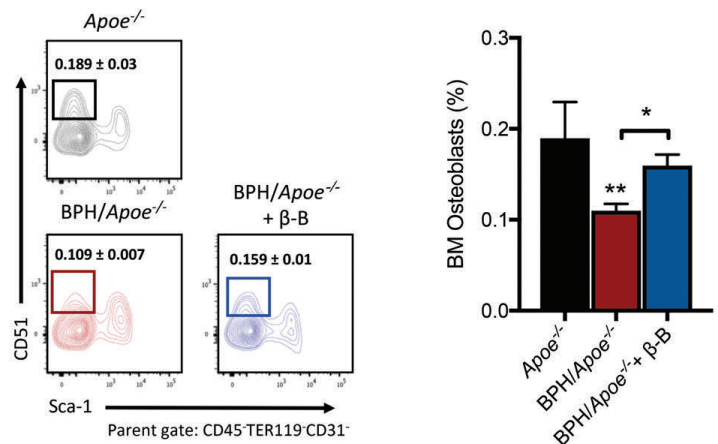

H \& E

$\mathbf{F}$

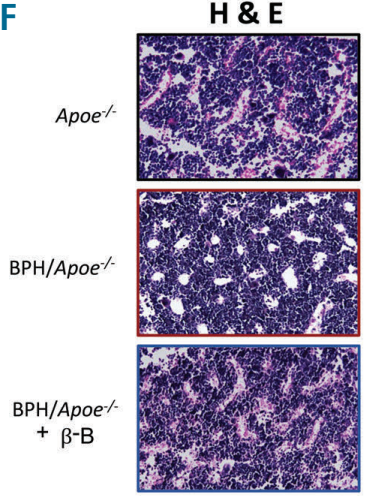

G
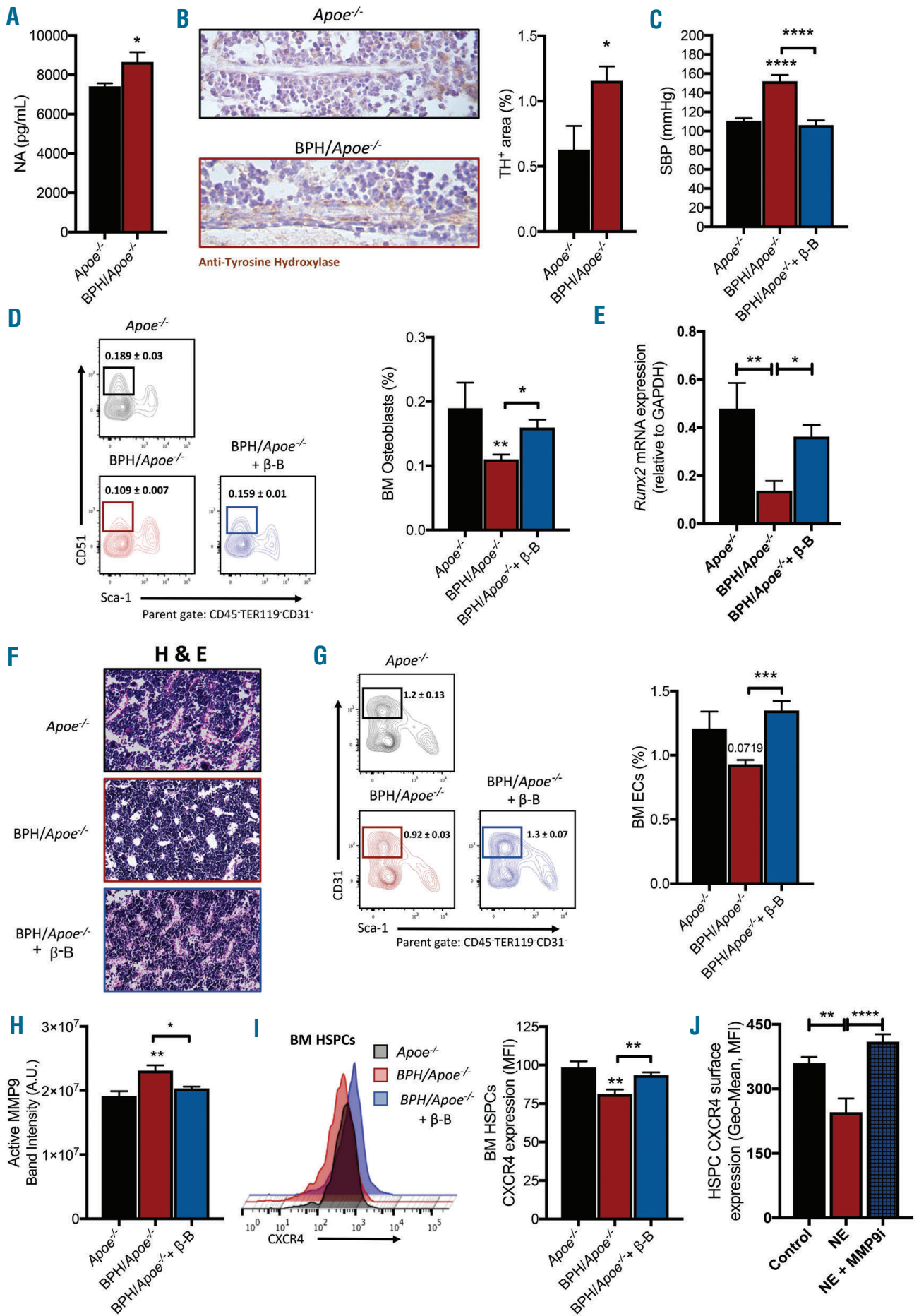

Figure 4. Sympathetic nervous system signalling induces a breakdown of the HSPC niche in the BM. Apoe $\%$ and $\mathrm{BPH} / \mathrm{Apoe}^{*}$ mice were fed a WTD for 16 weeks with $\mathrm{BPH} / \mathrm{Apoe}^{\%}$ mice either treated with or without Propranolol $(0.5 \mathrm{~g} / \mathrm{L})$ in drinking water. A) Plasma NA was quantified by HPLC. B) BM sections were immunostained for sympathetic activity as indicated by tyrosine hydroxylase, imaged at X20; scale bar $=25 \mu \mathrm{m}$. C) Systolic blood pressure of mice following 16 weeks treatment. D) Osteoblastic lineage cells were quantified by flow cytometry. E) mRNA levels of Runx2 in the BM. F) H\&E stained representation of $B M$ vascular morphology, imaged at X10 scale bar $=100 \mu \mathrm{m}$. G) BMECs were measured by flow cytometry. H) MMP9 content in the BM extracellular fluid was determined via Zymography. I) CXCR4 expression levels on HSPCs was assessed by flow cytometry from and J) neutrophil supernatant cultured HSPCs. Data are presented as mean \pm SEM where $* P<0.05$ $* * P<0.01, \quad * * * P<0.001$, $* * * * P<0.0001$ (Student's $t$ test or One-Way ANOVA). A) $n=$ 8, B) $n=5$, C) $n=7$, D) $n=7-9$, E) $\mathrm{n}=5-9$, G) $\mathrm{n}=7-9$, H) $5-9$, I) $\mathrm{n}=$ $12-15$, J) $n=7$. 


\section{$\mathrm{BPH} / \mathrm{Apoe}^{-/-}$mice have enhanced myelopoiesis}

An overactive SNS has recently been shown to promote the mobilization of BM HSPCs to the spleen, resulting in the generation of splenic monocytes that can infiltrate into atherosclerotic lesions. Therefore, we next assessed the hematopoietic system in these mice. ${ }^{21}$ We discovered prominent monocytosis and neutrophilia in the $\mathrm{BPH} /$ Apoe $^{-1}$ mice in the blood (Figure 3A). Next, to determine if the increased monocyte and neutrophil numbers were due to activated myelopoiesis, we examined the abundance and proliferation of HSPCs and myeloid progenitor cells in the BM. While the levels and proliferation of HSPCs within the BM were similar (Figure 3B, C), we did observe more granulocyte-macrophage progenitors (GMPs) in the BPH/Apoe mice, which were proliferating at a higher rate (Figure 3D,E). Consistent with SNS activation in promoting the mobilisation of HSPCs from the $\mathrm{BM}$, we detected elevated levels of circulating HSPCs and myeloid progenitors (MPCs) in the BPH/Apoe mice (Figure 3F). Given the higher circulating HSPCs, we were expecting to see more HSPCs in the spleen. However, no such change in the abundance of HSPCs was detected (Figure 3G). Of note, a higher proportion were in the G2M phase of the cell cycle (Figure $3 \mathrm{H}$ ), suggesting that the chronic activation of the SNS was influencing the HSPCs to proliferate more in the spleens of $\mathrm{BPH} / \mathrm{Apoe}^{-1}$ mice. Indeed, monocytes and neutrophils were elevated in the spleens of the BPH/Apoe ${ }^{-/}$mice, confirming extramedullary myelopoiesis was occurring in this chronic sympathetic driven model (Figure 3I).

\section{Sympathetic activation contributes to the breakdown of the HSPC bone marrow microenvironment}

The Schlager mice are an established model of sympathetic activation-mediated hypertension. ${ }^{26}$ However, we wanted to confirm that there was evidence of increased sympathetic activation in the $\mathrm{BM}$, which could account for the enhanced mobilization of HSPCs observed in Figure 3D. Firstly, to confirm an overall increase in sympathetic tone, we quantified plasma noradrenaline levels, which we found to be higher in the BPH/Apoe mice (Figure 4A). More central to our proposed mechanism for enhanced HSPC mobilization in the BPH/Apoe-- mice, we found enhanced expression of tyrosine hydroxylase (TH), the rate-limiting enzyme found in nerve terminals responsible for noradrenaline (NA) production, around the blood vessels in the $\mathrm{BM}$ of the $\mathrm{BPH} / \mathrm{Apoe}^{-1}$ mice (Figure $4 \mathrm{~B}$ ). Together, these data reveal a more global increase in sympathetic tone in SNS-driven hypertension.

Next, we sought to determine if overactive sympathetic signaling in the $\mathrm{BM}$ led to changes within the $\mathrm{BM}$ microenvironment and whether these changes could be reversed with the use of a $\beta$-blocker. Given the importance of sympathetic overdrive in mediating hypertension, as expected, propranolol normalized blood pressure in the $\mathrm{BPH} /$ Apoe $^{-/}$mice (Figure $4 \mathrm{C}$ ).

Having demonstrated that propranolol could reverse the systemic responsiveness of $\beta$-receptors to sympathetic activation in $\mathrm{BPH} / \mathrm{Apoe}^{-1}$ mice, we examined key HSPC niche cells in the BM to determine if sympathetic overdrive influenced myelopoiesis via effects on the BM niche. Interestingly, we found a significant reduction in the abundance of $\mathrm{CD} 51^{+}$osteoblasts in the $\mathrm{BM}$ on the $\mathrm{BPH} / \mathrm{Apoe}^{-1}$ mice, which were restored when these mice were treated with propranolol (Figure 4D). Consistent with this find- ing, analysis of BM mRNA for Runx2, the transcription factor that drives osteoblast production, showed a reduction in Runx2 expression in the $\mathrm{BPH} / \mathrm{Apoe}^{-1}$ mice relative to Apoe $e^{-/}$mice. Similar to our flow cytometry data, treatment with propranolol prevented the suppression of Runx2 expression (Figure 4E). When we assessed the gross morphological changes in the $\mathrm{BM}$, it appeared that the vascular structures were altered with the $\mathrm{BPH} / \mathrm{Apoe}^{-1}$ mice showing smaller sinusoidal structures relative to the Apoe mice, with propranolol reverting the sinusoids back to that seen in the Apoe mice (Figure 4F). Furthermore, in examining the endothelial cell population, we noted a trend towards a decrease in the abundance of these cells, which, again, could be restored with the administration of propranolol (Figure 4G). As these niche cells are an important source of the HSPC retention factor CXCL12, we measured its mRNA expression and found that propranolol greatly increased Cxcl12 expression, thereby potentially aiding in promoting HSPC retention and reduced quiescence in the BM (Online Supplementary Figure S2, A). The changes in these two key niche cells may provide a mechanism for increased HSPC release from the BM in $\mathrm{BPH} /$ Apoe $^{-1}$ mice. Other cells within the $\mathrm{BM}$ express $\beta$-adrenoreceptors, which we profiled using gene array data from Novershtern et al. and analysed using online software (BloodSpot) to generate a hierarchical differentiation tree. ${ }^{2,30}$ Firstly, HSPCs and myeloid progenitors did not display any enrichment for the adrenoceptors. However, neutrophils were identified as one of the cells enriched in transcripts for the $\beta 2$-adrenoreceptor, but not $\beta 1$ - or $\beta 3$-adrenoreceptors (Online Supplementary Figure S2, $B-D)$. We pharmacologically confirmed the requirement for $\beta 2$-adrenoreceptor stimulation in HSPC mobilization using the BPH mice on an Apoe $e^{++}$background by administering the $\beta 2$ specific antagonist ICI-118551 (Online Supplementary Figure S2, E). Furthermore, neutrophils have previously been shown to be responsive to NA in vitro. ${ }^{31,32}$ Mechanistically, activated neutrophils can release MMP9 which can cleave CXCR4 on HSPCs, providing another avenue to HSPC liberation from the BM. ${ }^{22,33}$ We measured levels of MMP9 in the BM extracellular fluid (BMEF) via zymography and found that both active and latent MMP9 levels increased in the $\mathrm{BPH} / \mathrm{Apoe}^{-1}$ mice, a phenotype reversed with propranolol treatment (Figure $4 \mathrm{H}$ and Online Supplementary Figure S2, F). In support of this we found reduced surface CXCR4 expression on the HSPCs from the $\mathrm{BPH} / \mathrm{Apoe}^{-1}$ mice, which was restored in mice treated with propranolol (Figure 4I). These data were further supported by BM mRNA analysis indicating that propranolol treatment increases Cxcr4 expression (Online Supplementary Figure S2, G). To explore this mechanism further, we cultured HSPCs in the supernatants of neutrophils treated with NA and examined CXCR4 cell surface abundance. We found significantly less CXCR4 on HSPCs cultured in supernatants from NA activated neutrophils (isolated from wild-type mice), compared to vehicle treated neutrophils, which was prevented when MMP9 was inhibited (Figure 4J). When we included the $\beta 2$-adrenoreceptor specific inhibitor ICI-118551 into the BM neutrophil stimulation media with NA, the harvested supernatant caused less efficient cleavage of CXCR4 (Online Supplementary Figure S2, H) thereby confirming the role for neutrophil $\beta 2$-adrenoreceptors. These data support the hypothesis that sympathetic activation is present in the $\mathrm{BM}$ of the $\mathrm{BPH} / \mathrm{Apoe}^{-1}$ mice and responsible for the 
mobilization of HSPCs by acting on key niche cells along with stimulating neutrophils to secrete proteases that cleave the retention receptor CXCR4 on HSPCs.

\section{Suppressing chronic sympathetic signaling dampens myelopoiesis in hypertensive $\mathrm{Apoe}^{-/}$mice}

Having observed a restoration in the HSPC BM microenvironment when $\mathrm{BPH} / A$ poe $e^{-1}$ mice were treated with propranolol, we explored if this was also reflected by normalization of myelopoiesis in these mice. Following treatment with propranolol, we observed a reduction in circulating monocytes and neutrophils (Figure 5A). We determined if this reduction was echoed by changes in the BM stem and progenitor populations. Following administration of propranolol, the abundance of BM HSPCs was not affected; however, these cells were proliferating at lower rates and giving rise to fewer GMPs (Figure 5, B-D). Consistent with an improvement in the HSPC microenvironment, there were fewer mobilized HSPCs and MPCs in the blood of the $\mathrm{BPH} /$ Apoe $^{-1}$ mice treated with propra-
A

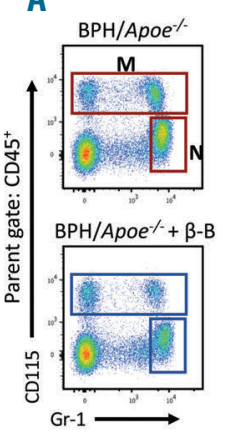

D

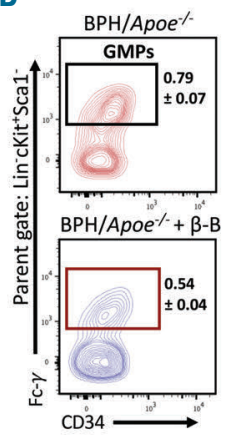

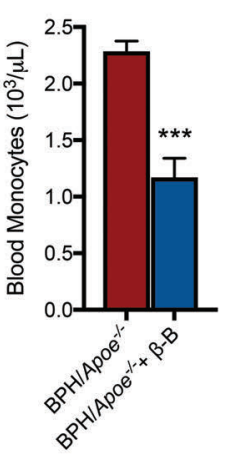
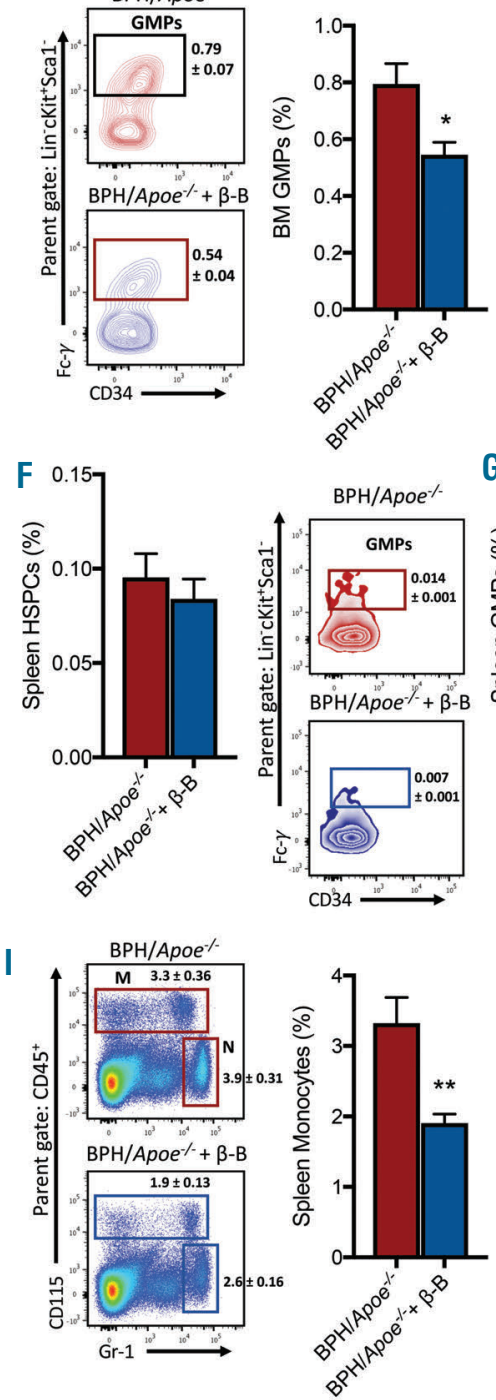

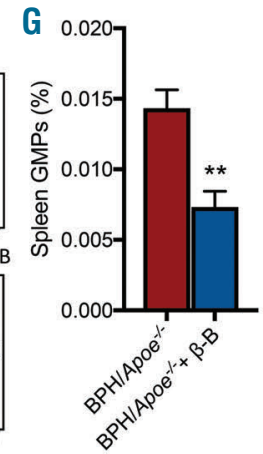

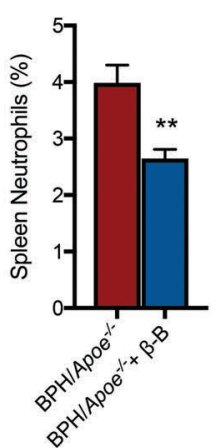

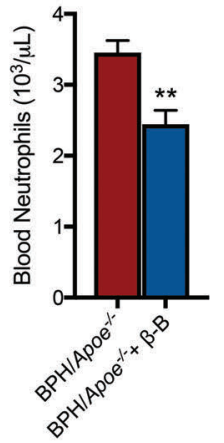

$\mathrm{E}$
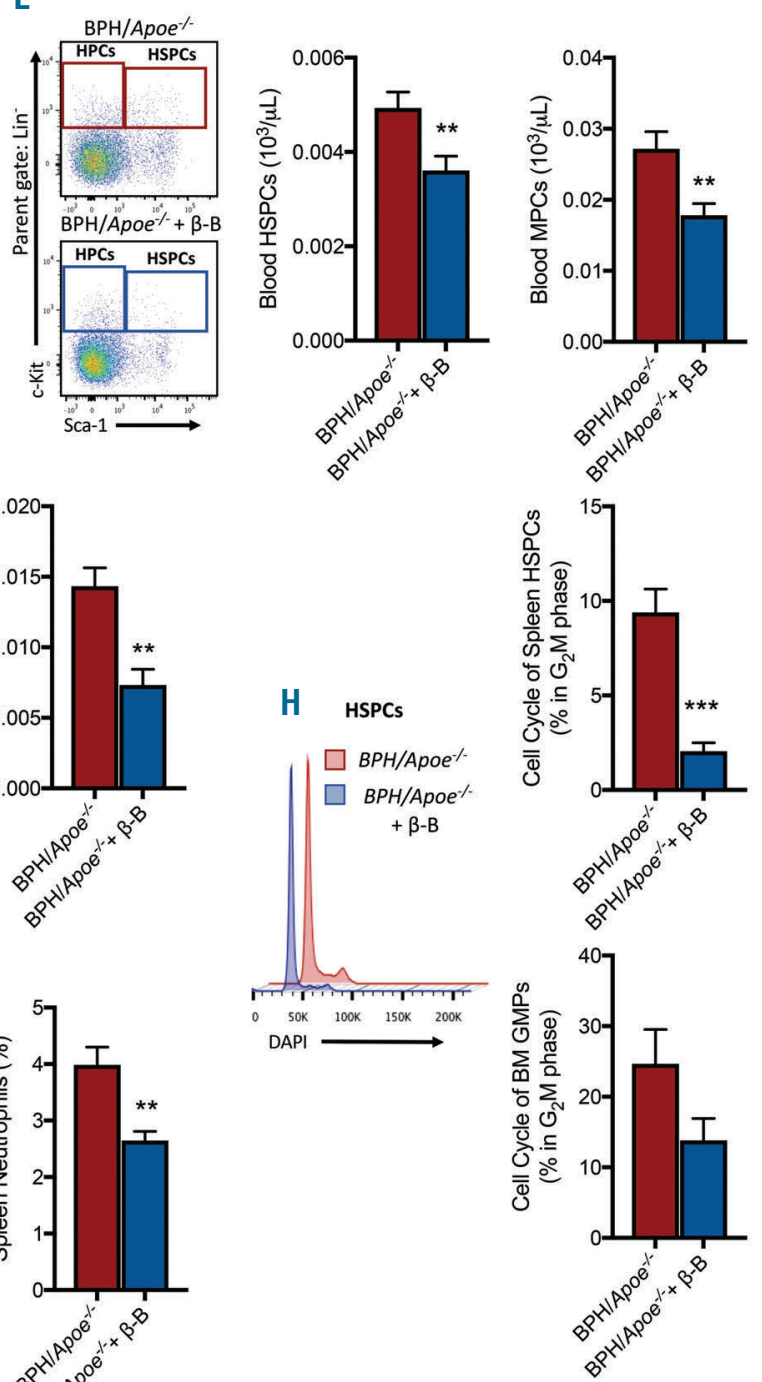

Figure 5. Propranolol prevents extramedullary hematopoiesis in $\mathrm{BPH} / \mathrm{Apoe}$ - mice. BPH/Apoe mice were fed a WTD for 16 weeks and treated with vehicle or Propranolol $(0.5 \mathrm{~g} / \mathrm{L})$ in drinking water. Flow cytometry was used to assess A) circulating monocytes(M)/neutrophils(N), B) BM HSPCs and C) proportion of which are proliferating along with $D$ ) of $B M$ GMPs. E) Blood HSPC and MPC populations. F) Spleen HSPC and G) GMP populations along with $H$ ) proliferating splenic HSPCs and GMPs were assessed by flow cytometry. I) Splenic monocyte(M)/neutrophil(N) populations. Data are presented as mean \pm SEM where $* P<0.05, * * P<0.01$ and $* * * P<0.001$ (Student's $t$-test). $\mathrm{A}-\mathrm{I}) \mathrm{n}=6-10$. 
nolol (Figure 5E). This was paralleled by a decrease in extramedullary hematopoiesis in the spleen as evidenced by fewer proliferating HSPCs, GMPs and less monocytes and neutrophils (Figure 5, F-I). Taken together, these data suggest that lowering responsiveness to chronic sympathetic signaling in the $\mathrm{BPH} / \mathrm{Apoe}^{-/}$mice results in an overall dampening of myelopoiesis.

\section{Blocking sympathetic signalling decreases} atherosclerosis in $\mathrm{BPH} / \mathrm{Apoe}^{-/-}$mice

To examine if the reduction in sympathetic tone and dampening of myelopoiesis was associated with reduced atherosclerotic plaque progression, we assessed the size and complexity of lesions in the proximal aorta. Firstly, we noted a reduction in lesion size in the proximal aorta and aortic arch of $\mathrm{BPH} / \mathrm{Apoe}^{-/}$mice treated with propranolol (Figure 6A, B). Exploring the lesion characteristics, we noted that propranolol treated mice had reduced plaque lipid accumulation along with a reduction in plaque macrophages (Figure 6C, D). We also observed a trend for increased collagen (Figure 6E). These changes were seen in the absence of any changes in plasma cholesterol levels (Figure 6F). Given that the hypertension in the BPH/Apoe/- mice did not promote endothelial dysfunction, it sug-
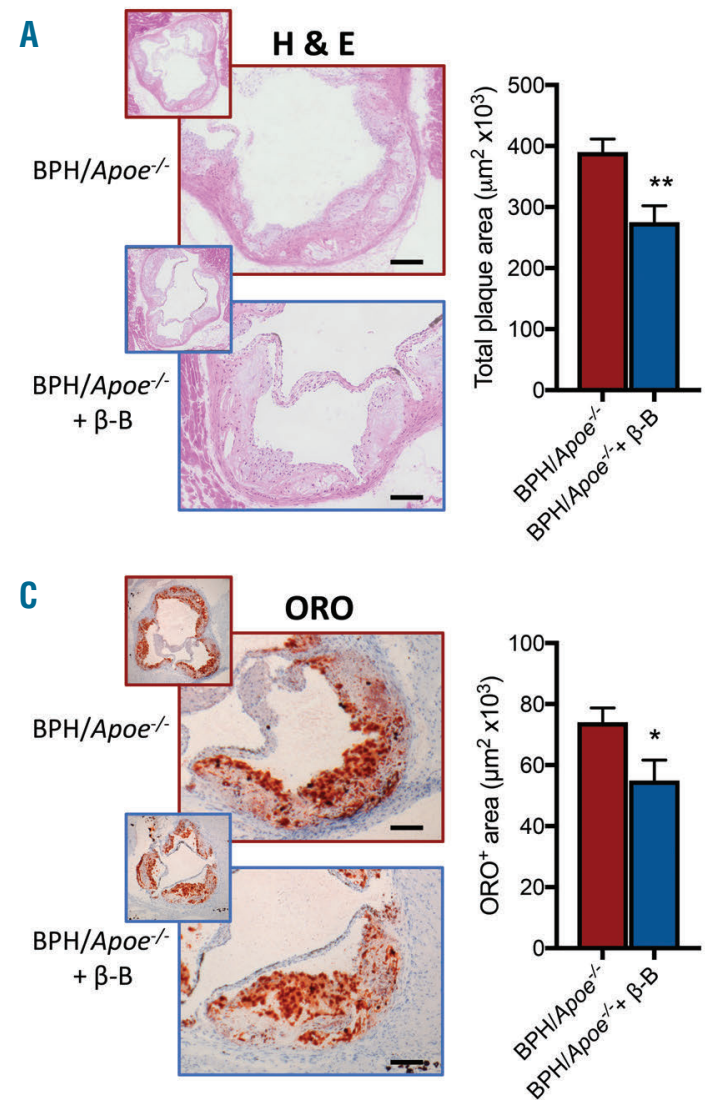

E
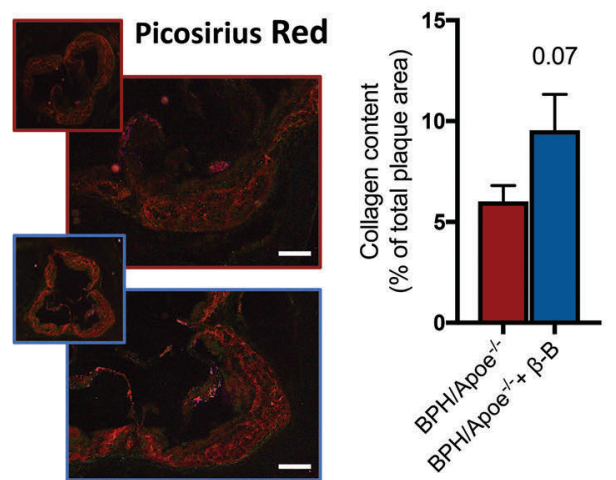

B

\section{Aortic Arch ORO}

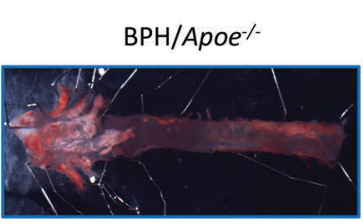

$\mathrm{BPH} / \mathrm{Apoe}^{-/}+\beta-\mathrm{B}$
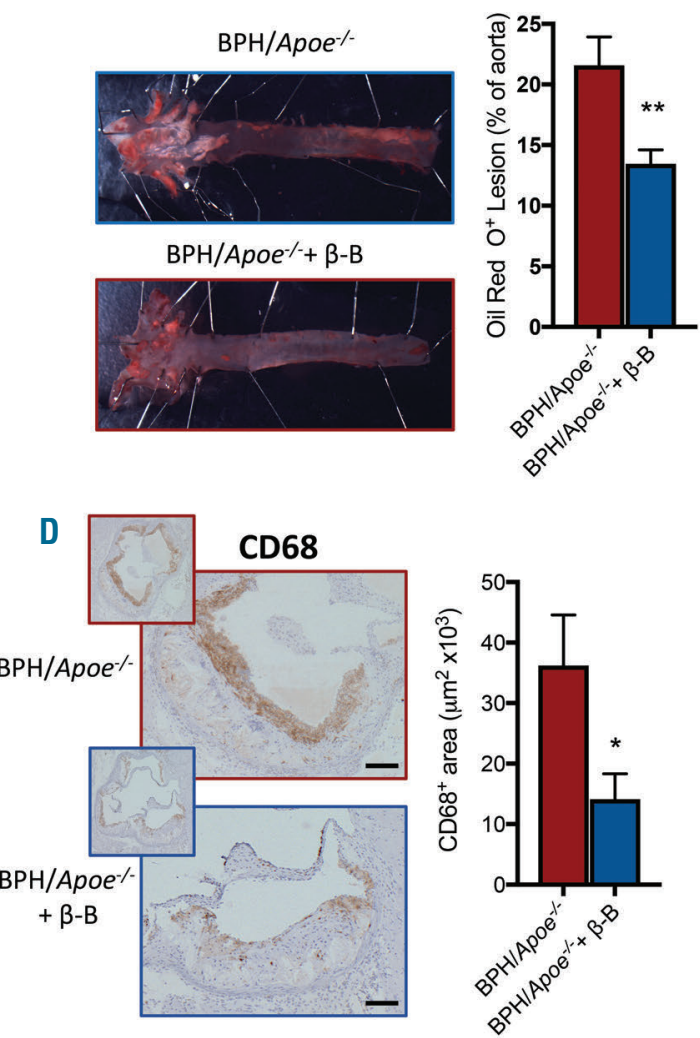

F

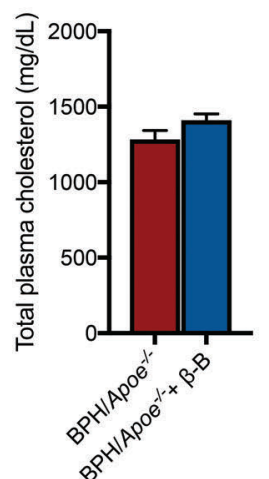

Figure 6. Propranolol inhibits plaque progression in $\mathrm{BPH} / \mathrm{Apoe}^{\%}$ mice. BPH/Apoe ${ }^{\%}$ mice were fed a WTD for 16 weeks and treated with vehicle or Propranolo $(0.5 \mathrm{~g} / \mathrm{L})$ in drinking water. At the end point, atherosclerosis in the proximal aorta was assessed for A) H\&E staining for plaque size in the proximal aorta and B) lipid content $(\mathrm{ORO}+)$ lesions in the aortic arch were quantified. Proximal aortas were also stained for $\mathrm{C}$ ) lipid content (ORO), D) macrophages (CD68) and E) collagen (picosirius red). Lesions were imaged at X4 (insets) and zoomed in to view single lesion, scale bar $=100 \mu \mathrm{m}$. F) Total plasma cholesterol levels. Data are presented as mean \pm SEM where $* P<0.05$ and $* * P<0.01$ (Student's $t$-test). A,B) $n=9, C$-E) $n=7, F) n=9$. 
gests that the improvements in plaque size and complexity are due to dampened myelopoiesis and, subsequently, reduced monocyte infiltration.

\section{Discussion}

Chronic hypertension is arguably one of the most common risk factors associated with atherosclerotic CVD. ${ }^{1}$ However, delving into the responsible mechanism(s), it remains unclear if an increase in blood pressure alone, or in conjunction with a change in concurrent signaling events such activation of the RAS or sympathetic activation, directly contributes to atherosclerotic CVD. Using a genetic model of hypertension driven by sympathetic activation, we show that this form of hypertension (compared to atherosclerotic prone mice without hypertension) alters the characteristics of the atherosclerotic lesion to a more unstable phenotype, hallmarked by increased macrophage accumulation. We also found that chronic sympathetic activation caused changes in hematopoiesis. In particular, increased sympathetic activity was found in the $\mathrm{BM}$, altering the HSPC microenvironment and causing the liberation of certain stem cells to the spleen where monocytes were generated. This was accompanied by an increase in blood monocytes, likely explaining the increased macrophage burden observed in the atherosclerotic lesions. These atherogenic pathways could all be inhibited pharmacologically by blocking sympathetic signalling through $\beta$-adrenoreceptors using propranolol. These findings suggest that chronic sympathetic activation, present in many forms of hypertension, likely contributes to the increased CVD risk by modulating hematopoiesis, independent of endothelial dysfunction.

With respect to understanding the contribution of hypertension to vascular disease, the majority of research has focused on the effects on the endothelium. Perhaps the most common belief is that hypertension causes endothelial dysfunction and activation, which in turn recruits immune cells and forms the main mechanism propagating the atheroma. Interestingly, we found no evidence of endothelial dysfunction in our hypertensive mice, at least in this model of a dominant sympathetic driven form of hypertension, the endothelial dysfunction was not contributing significantly to atherogenisis. ${ }^{34}$ Supporting our theory that underlying sympathetic nervous signaling, that may be independent of pressure itself, can drive atherogenesis, moderate increases in AngII are sufficient to promote accelerated atherogenesis, without elevations in blood pressure. ${ }^{3,4}$ Additionally, AngII has also been shown to invoke a T-helper cell (TH1) immune response to promote atherogenesis independent of its hemodynamic effects. Thus, signaling events that can cause hypertension are likely important in driving CVD through their immune modulatory responses. ${ }^{7-10,35,36}$ Further, with the discovery of accelerated vascular disease driven by acute events triggering sympathetic activation leading to enhanced monocyte production, it is plausible that this pathway is triggered in chronic SNS-driven hypertension and would contribute to accelerated atherosclerosis. ${ }^{21,23,24}$ We hypothesized that the overactive SNS seen in subgroups of patients with hypertension would contribute to atherogenesis by stimulating hematopoiesis. Importantly, elevated WBCs are associated with the incidence of hypertension and predicts CV outcomes in this patient group. ${ }^{37-39}$ However, the cause of increased WBCs in hypertensive patients has not been resolved.

Consistent with recent studies which have observed monocytosis following acute scenarios of sympathetic activation, we too observed monocytosis in the hypertensive $\mathrm{BPH} /$ Apoe $^{-/}$mice. ${ }^{21,24}$ The initial predominant change driven by the overactive sympathetic signaling in our study, relevant to increased myelopoiesis, appears to occur within the BM. We noted a decreased abundance of two key niche cells, endothelial cells and osteoblasts, which harbour anchoring points in the marrow for HSPCs, preventing their release into circulation. ${ }^{40-43}$ The contribution of the SNS in regulating this process was first described by a seminal study from the Frenette laboratory, detailing the requirement of a functional SNS in the BM, which is required for G-CSF mediated HSPC mobilization. ${ }^{16}$ Almost a decade later, the Nahrendorf group discovered the importance of this pathway in respect to CVD, revealing that sympathetic activation following an acute myocardial infarction promotes HSPC liberation to the spleen where the production of an additional atherogenic pool of monocytes occurs. ${ }^{21}$ The absence of an expanded HSPC population in the spleen is likely due to the chronic nature of our study and suggests that these cells likely rapidly matured into myeloid committed progenitors. The recent studies then suggest that the monocytes generated, migrated into the atherosclerotic lesion, and enhanced macrophage burden, potentiating the risk of a secondary CV event. Our data reveal that this process is occurring chronically and identifies an important mechanism that likely contributes to atherogenesis and the increased risk of a CV event in hypertension. We also identified another pathway by which sympathetic signaling can induce the liberation of BM HSPCS by causing a decrease in the HSPC-expressed retention receptor CXCR4. Given that HSPCs do not appear to express $\beta$ adrenoreceptors, it suggested a cell extrinsic mechanism resulting in less HSPC cell surface CXCR4. Interestingly, neutrophils express $\beta 2$ adrenoceptors and can be activated after sensing NE (Online Supplementary Figure S2, B-C). Modelling this in vitro revealed that NE-activated neutrophils produce MMP9, which cleaves CXCR4 on HSPCs. Thus, BM sympathetic activation likely liberates HSPCs via multiple mechanisms, some of which are independent of the previously described SNS/G-CSF axis.

As mentioned above, there are several studies that have identified a role for $\beta$ adrenoreceptors in influencing HSPC release via modulating the $\mathrm{BM}$ niche. There is strong evidence for the role of $\beta-3$ adrenergic receptor in regulating nestin+ stromal cell production of key factors such as CXCL12, angiopoietin and stem cell factor, thereby influencing HSPC retention and proliferation. $\beta-3$ antagonism following ischemic events has shown reduced HSPC mobilisation and proliferation leading to dampened extramedullary hematopoiesis. ${ }^{19,21,23,24}$ However, there is also strong evidence pointing to a role for the $\beta-2$ adrenergic receptor in regulating $\mathrm{BM}$ niche components and HSPC mobilisation. Although this has been suggested to occur through other niche components such as osteoblasts and other stromal cells and not specifically nestin+ cells. ${ }^{16,18}$ These findings regarding the role of $\beta$ adrenoceptors in modulating HSPC mobilisation suggest that in our study there is a likely contribution of both $\beta-2$ and $\beta-3$ receptors to changes in the BM. However, considering the role of $\beta-2$ in the setting of hypertension and elevated 
blood pressure we focused on the specific role of $\beta-2$. Interestingly, a recent study by Mendez-Ferrer's group has also highlighted the chronic role of nestin ${ }^{+}$cells present in the BM and other tissues in regulating myeloid cell movement in the setting of atherosclerosis. ${ }^{44}$ Given that nestin ${ }^{+}$ cells in the BM express $\beta-3$ receptors and the data we have presented above regarding chronic sympathetic driven hypertension and its contribution to atherosclerosis, it is likely that this pathway may also play a role and warrants further investigation.

The obvious limitation of this study is that our findings were generated in mice. However, this also allowed us to isolate a prominent form of hypertension to reveal a novel atherogenic mechanism, which appears to be independent of endothelial dysfunction, and thus our findings also permit the current dogma to be challenged. While we revealed the effectiveness of propranolol in this model, there is a need to further investigate the effects of directly reducing enhanced hematopoiesis without targeting systemic blood pressure. It is likely that with the development of antiinflammatory drugs targeted at the hematopoietic system, it would be possible to dampen the effects on the hematopoietic system without affecting blood pressure which would hypothetically provide the same conclusions as in the present study. Finally, we only studied one form of hypertension, driven by sympathetic signaling. It would be of specific importance to extend a modified version of this hypothesis to hypertension driven by the RAS.

\section{Funding}

AJM is Career Development Fellow of the NHMRC (APP1085752) and a Future Leader Fellowship from the National Heart Foundation (100440) and a recipient of a CSL Centenary Award. This study was also supported by NHMRC project grants (APP1106154 and APP1142938) to AJM. and J.C-D. M.J.K is a Russell Berrie Foundation Scholar in Diabetes Research from the Naomi Berrie Diabetes Centre. PRN was supported by grants from the NIH (R01HL1379 \& ROOHL1225).

\section{References}

1. (2015) WHO. Non Communicable diseases in Health in 2015: From MDG to SDG. Contract No: ISBN 978954156511. 2015;

2. Bondjers G, Glukhova M, Hansson GK, Postnov YV, Reidy MA, Schwartz SM. Hypertension and atherosclerosis. Cause and effect, or two effects with one unknown cause? Circulation. 1991;84(6 Suppl):VI2-16.

3. Daugherty A, Manning MW, Cassis LA. Angiotensin II promotes atherosclerotic lesions and aneurysms in apolipoprotein Edeficient mice. J Clin Invest. 2000;105(11):1605-1612.

4. Mazzolai L, Duchosal MA, Korber M, et al. Endogenous angiotensin II induces atherosclerotic plaque vulnerability and elicits a Th1 response in ApoE-/- mice. Hypertension. 2004;44(3):277-282.

5. Noll G, Wenzel RR, Binggeli C, Corti C, Luscher TF. Role of sympathetic nervous system in hypertension and effects of cardiovascular drugs. Eur Heart J. 1998;19 Suppl F:F32-38.

6. Esler M, Jennings G, Korner $\mathrm{P}$, et al. Assessment of human sympathetic nervous system activity from measurements of norepinephrine turnover. Hypertension. 1988; 11(1):3-20.

7. Zubcevic J, Jun JY, Kim S, et al. Altered inflammatory response is associated with an impaired autonomic input to the bone marrow in the spontaneously hypertensive rat. Hypertension. 2014;63(3):542-550.

8. Santisteban MM, Zubcevic J, Baekey DM, Raizada MK. Dysfunctional brain-bone marrow communication: a paradigm shift in the pathophysiology of hypertension. Curr Hypertens Rep. 2013;15(4):377-389.

9. Santisteban MM, Kim S, Pepine CJ, Raizada MK. Brain-gut-bone marrow axis: implications for hypertension and related therapeutics. Circ Res. 2016;118(8):1327-1336.

10. Santisteban MM, Ahmari N, Carvajal JM, et al. Involvement of bone marrow cells and neuroinflammation in hypertension. Circ Res. 2015;117(2):178-191.
11. Ross R. Atherosclerosis is an inflammatory disease. Am Heart J. 1999;138(5 Pt 2):S419 420.

12. Woollard KJ, Geissmann F. Monocytes in atherosclerosis: subsets and functions. Nat Rev Cardiol. 2010;7(2):77-86.

13. Murphy AJ, Tall AR. Disordered haematopoiesis and athero-thrombosis. Eur Heart J. 2016;37(14):1113-1121

14. Qiao JH, Tripathi J, Mishra NK, et al. Role of macrophage colony-stimulating factor in atherosclerosis: studies of osteopetrotic mice. Am J Pathol. 1997;150(5):1687-1699.

15. van der Valk FM, Kuijk C, Verweij SL, et al Increased haematopoietic activity in patients with atherosclerosis. Eur Heart J. 2017;38(6):425-432.

16. Katayama Y, Battista M, Kao WM, et al Signals from the sympathetic nervous system regulate hematopoietic stem cell egress from bone marrow. Cell. 2006;124(2):407421.

17. Stiekema LCA, Schnitzler JG, Nahrendorf $M$. Stroes ESG. The maturation of a 'neural-hematopoietic' inflammatory axis in cardiovascular disease. Curr Opin Lipidol. 2017;28(6):507-512.

18. Mendez-Ferrer S, Battista M, Frenette PS Cooperation of beta(2)- and beta(3)-adrenergic receptors in hematopoietic progenitor cell mobilization. Ann N Y Acad Sci. 2010; 1192:139-144.

19. Mendez-Ferrer S, Lucas D, Battista M Frenette PS. Haematopoietic stem cell release is regulated by circadian oscillations. Nature. 2008;452(7186):442-447.

20. Robbins CS, Chudnovskiy A, Rauch PJ, et al. Extramedullary hematopoiesis generates Ly-6C(high) monocytes that infiltrate ath erosclerotic lesions. Circulation. 2012;125(2):364-374.

21. Dutta P, Courties G, Wei Y, et al Myocardial infarction accelerates atherosclerosis. Nature. 2012;487(7407):325-329.

22. Westerterp M Gourion-Arsiquaud S Murphy AJ, et al. Regulation of hematopoietic stem and progenitor cell mobilization by cholesterol efflux pathways. Cell Stem Cell. 2012;11(2):195-206.

23. Heidt T, Sager HB, Courties G, et al
Chronic variable stress activates hematopoietic stem cells. Nat Med. 2014 20(7):754-758.

24. Courties G, Herisson F, Sager HB, et al Ischemic stroke activates hematopoietic bone marrow stem cells. Circ Res. 2015; 116(3):407-417

25. Esler M, Jennings G, Lambert G. Measurement of overall and cardiac norepinephrine release into plasma during cognitive challenge. Psychoneuroendocrinology. 1989;14(6):477-481.

26. Davern PJ, Nguyen-Huu TP, La Greca L, Abdelkader A, Head GA. Role of the sympathetic nervous system in Schlager genetically hypertensive mice. Hypertension. 2009;54(4):852-859.

27. Brandes RP. Endothelial dysfunction and hypertension. Hypertension. 2014;64(5): 924-928.

28. Guzik TJ, Hoch NE, Brown KA, et al. Role of the $\mathrm{T}$ cell in the genesis of angiotensin II induced hypertension and vascular dysfunction. J Exp Med. 2007;204(10):2449. 2460

29. Novershtern N, Subramanian A, Lawton LN, et al. Densely interconnected transcriptional circuits control cell states in human hematopoiesis. Cell. 2011;144(2):296-309.

30. Bagger FO, Sasivarevic D, Sohi SH, et al BloodSpot: a database of gene expression profiles and transcriptional programs for healthy and malignant haematopoiesis. Nucleic Acids Res. 2016;44(D1):D917924.

31. Kim MH, Gorouhi F, Ramirez S, et al Catecholamine stress alters neutrophil trafficking and impairs wound healing by beta2-adrenergic receptor-mediated upregulation of IL-6. J Invest Dermatol. 2014; 134(3):809-817.

32. Nicholls AJ, Wen SW, Hall P, and MJH, Wong CHY. Activation of the sympathetic nervous system modulates 1 neutrophil function. J Leukoc Biol. 2018;103(2):295309 .

33. Levesque JP, Hendy J, Takamatsu Y, Simmons PJ, Bendall LJ. Disruption of the CXCR4/CXCL12 chemotactic interaction during hematopoietic stem cell mobiliza- 
tion induced by GCSF or cyclophosphamide. J Clin Invest. 2003;111(2):187196.

34. Susic D. Hypertension, aging, and atherosclerosis. The endothelial interface. Med Clin North Am. 1997;81(5):1231-1240.

35. Zubcevic J, Santisteban MM, Pitts T, et al. Functional neural-bone marrow pathways: implications in hypertension and cardiovascular disease. Hypertension. 2014; 63(6):e129-139.

36. Wei Z, Spizzo I, Diep H, Drummond GR, Widdop RE, Vinh A. Differential phenotypes of tissue-infiltrating $\mathrm{T}$ cells during angiotensin II-induced hypertension in mice. PloS One. 2014;9(12):e114895

37. Schillaci G, Pirro M, Pucci G, et al.
Prognostic value of elevated white blood cell count in hypertension. Am J Hypertens. 2007;20(4):364-369.

38. Karthikeyan VJ, Lip GY. White blood cell count and hypertension. J Hum Hypertens. 2006;20(5):310-312.

39. Nakanishi N, Sato M, Shirai K, Suzuki K Tatara K. White blood cell count as a risk factor for hypertension; a study of Japanese male office workers. J Hypertens. 2002; 20(5):851-857.

40. Calvi LM, Adams GB, Weibrecht KW, et al. Osteoblastic cells regulate the haematopoietic stem cell niche. Nature. 2003; 425(6960):841-846.

41. Semerad CL, Christopher MJ, Liu F, et al. G-CSF potently inhibits osteoblast activity and CXCL12 mRNA expression in the bone marrow. Blood. 2005;106(9):3020 3027.

42. Hooper AT, Butler JM, Nolan DJ, et al. Engraftment and reconstitution of hematopoiesis is dependent on VEGFR2mediated regeneration of sinusoida endothelial cells. Cell Stem Cell. 2009 4(3):263-274

43. Tamplin OJ, Durand EM, Carr LA, et al Hematopoietic stem cell arrival triggers dynamic remodeling of the perivascular niche. Cell. 2015;160(1-2):241-252.

44. Del Toro R, Chevre R, Rodriguez C, et al Nestin(+) cells direct inflammatory cell migration in atherosclerosis. Nat Commun. 2016;7:12706 